\title{
Favorable outcome in a patient with systemic BCGitis after intra-bladder instillation of Calmette-Guerin Bacillus highlighting the importance of making the correct diagnosis in this rare form of sepsis
}

\author{
Rachid Attou ${ }^{1}$, Thomas Albrich ${ }^{2}$, Joe Kadou ${ }^{2}$, Sebastien Redant ${ }^{1}$, \\ Patrick M. Honoré ${ }^{1}$, David De Bels ${ }^{1}$ \\ 'Intensive Care Department, Brugmann University Hospital, Brussels Belgium; \\ ${ }^{2}$ Students, Faculty of Medicine, Université Catholique de Louvain, Brussels, Belgium
}

Address for Correspondence: Prof. Patrick M Honoré, CHU-Brugmann University Hospital, Brussels, Belgium

E-mail: Patrick.Honore@CHU-

Brugmann.be

\begin{tabular}{|l|}
\hline Access this article online \\
\hline $\begin{array}{l}\text { Website: } \\
\text { www.intern-med.com }\end{array}$ \\
\hline DOI: \\
10.2478/jtim-2019-0007 \\
\hline Quick Response Code: \\
\hline \\
\hline \\
\hline
\end{tabular}

\section{ABSTRACT}

We present the case of a patient with sepsis following a traumatic intra-bladder instillation of Calmette-Guerin Bacillus with pneumonia and possibly hepatitis. These complications are rare and could be induced by both immuno-allergic reaction and bacteremia. There is no specific guideline to treat this condition, but many clinicians depicting similar cases seem to agree on prolonged anti-tuberculous antibiotics with associated corticosteroid therapy. Following this therapy, the prognosis is generally favorable depending upon the fact that diagnosis has correctly been made. Our case highlights the fact that correct diagnosis has to be made especially in the presence of sepsis without a clear septic source.

Key words: intra-bladder BCG instillation, BCG infection, pneumonia, hepatitis, sepsis, BCGitis

\section{INTRODUCTION}

Intra-bladder instillation of CalmetteGuerin Bacillus (BCG) is an immunostimulation and is used in the treatment of superficial in situ vesical carcinomas. BCG is considered as an adjuvant treatment after transurethral resection of superficial urothelial papillary carcinoma. ${ }^{[1]}$ This therapy does carry several risks. Of those, the most frequent risk is fever but local complication, such as a bladder trauma, is common. Nevertheless, systemic manifestations involving prostate, lung, liver and also kidney have been described. ${ }^{[2-3]}$

\section{CLINICAL CASE}

A 70-year old man, with a history of obstructive sleep apnea, type 2 diabetes, and hypertension was treated by BCG instillation for a bladder surface tumor. During the procedure, catheter placement was traumatic and hemorrhagic. Four hours later, the patient quickly deteriorated and was admitted to the emergency department. On admission, he had a flu-like syndrome with fever and chills. And he also suffered from oliguric acute kidney injury (AKI), liver failure, and thrombocytopenia including all the biologic signs of sepsis.

The patient was transferred to the intensive care unit (ICU) and amoxicillin-clavulanic acid was initiated. Blood testing highlighted an inflammatory syndrome [C-reactive protein (CRP) between 70 and $90 \mathrm{mg} / \mathrm{L}$, $<10]$ and hepatic cytolysis and cholestasis evoking liver involvement. Sediment and urinary cultures were negative. Blood cultures for mycobacteria were done after nightly fever peaks. Empirical anti-tuberculous treatment was started associating isoniazid, rifampicin and vitamin B6 in the event of a BCGitis. Chest X-Ray (Figure 1) showed an interstitial syndrome.

In spite of the introduction of this therapy, night fever peaks $\left(38-39^{\circ}\right.$ Centigrade (C)) persisted. Therefore, a primary source 


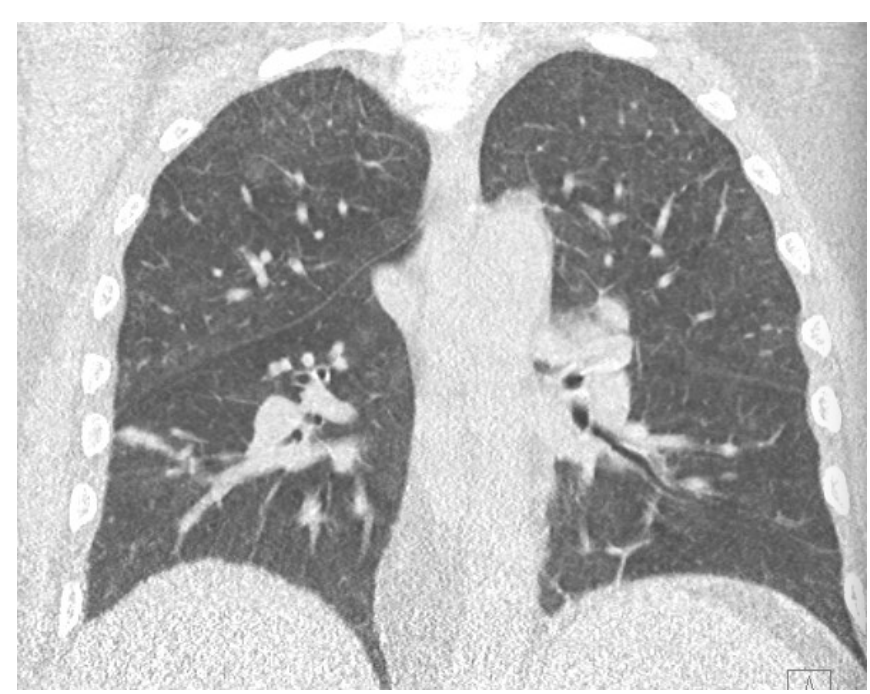

Figure 1: Chest X-ray

of sepsis was actively searched. On the first instance, prostatic encapsulated abscess secondary to the traumatic manipulation was investigated. Accordingly, antibiotic treatment was switched from amoxicillin-clavulanic acid to ceftazidime.

Aerobic and anaerobic blood cultures were all negative. Continuous investigation of the sepsis source encompasses positron emission tomography scanner (pet-scan), which revealed no infection site, and a pelvic magnetic resonance imagery, which excluded a prostatic abscess. After two weeks of therapy also, there was no improvement in the patient's condition (CRP remaining at $70 \mathrm{mg} / \mathrm{L}$ and liver enzymes increasing even more). Because of the liver deterioration, it was decided to stop every antimicrobial treatment allowing an open window in order to repeat cultures (blood, respiratory, and urinary tract).

At that time, the lung symptoms came with cough and grade II dyspnea with a bilateral pneumonia associated with pleural effusion on the lung tomodensitometry. A bronchoscopy together with a bronchoalveolar lavage (BAL) with Ziel and auramine colorations as well as trans-bronchial biopsies were performed. Four days later, the mycobacterial research came back negative and cytology highlighted inflammatory trails with the presence of granulocytes.

Having found no septic source, the possibility of a systemic BCGitis became more plausible. Accordingly, anti-tuberculous treatment was restarted, this time using isoniazid and moxifloxacin for 3 months associated with corticosteroids with a slow tapering down during a 3-month period.

After a week of this treatment, a definitive clinical and biological improvement was seen (no more fever or chills,

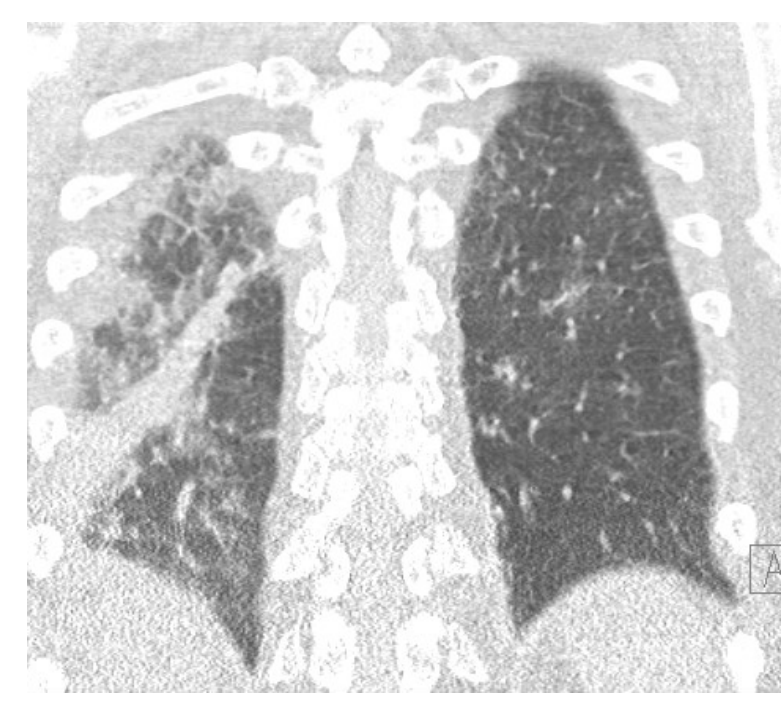

Figure 2: Thoracic tomodensitometry

normalization of inflammatory and hepatic markers, Table 1). Chest X-ray showed a regression of lung infiltrates. This tentative diagnosis was further confirmed and even definitively asserted with the presence of positive blood cultures 6 weeks later. Subsequently, the patient was moved to a rehabilitation ward before returning home.

\section{DISCUSSION}

In our clinical case, the patient presented a rapid complication of intra-bladder instillation of BCG, presenting as a flu-like syndrome. On admission, markers of sepsis were present. Despite a rapid classical antimicrobial therapy associated to a first line of anti-tuberculous drugs, the patient continued to have recurrent night fever associated with increasing biological inflammatory signs and rapidly evolving hepatitis. Two weeks after admission, the clinical condition worsened with signs of pleuro-pulmonary infection.

In spite the fact that BCG instillation is the reference treatment of proved superficial tumors, many side effects have been observed in the literature. ${ }^{[1-3]}$ First of all, the most common side effect is generally light and transitional (2-3 days) symptoms of local irritation of the bladder (90\%): cystitis, hematuria, dysuria, and pollakiuria. Systemic symptoms do occur too including flu, collapse, and fatigue that can start usually 4 hours after instillation and could persist for 24 to 48 hours.

If temperature is higher than $39^{\circ} \mathrm{C}$ despite antipyretic therapy and continues for more than 12 hours, a systemic BCGitis should be suspected associating local complications due to retrograde colonization of urinary track at different levels such as prostatic abscess or granulomatous prostatitis, epididymitis, renal abscess and glomerular hematuria. Systemic manifestations (3 to 5\%) 
Attou et al.: BCGitis after intra-bladder instillation of Calmette-Guerin Bacillus

\begin{tabular}{|c|c|c|c|c|c|}
\hline & $13 / 05$ & $15 / 05$ & $23 / 05$ & $06 / 06$ & $15 / 06$ \\
\hline & \multicolumn{5}{|c|}{ Admission } \\
\hline$\overline{\mathrm{Hb}}$ & 13 & 12.3 & 10.3 & 8.09 & 10.7 \\
\hline Neutrophils & 5.47 & 5.6 & 5.02 & 4.12 & 5.47 \\
\hline Lymphocytes & 0.08 & 0.23 & 0.58 & 0.55 & 1.09 \\
\hline Monocytes & 0.10 & 0.16 & 0.72 & 0.51 & 0.5 \\
\hline Platelets & 100 & 58 & 317 & 224 & 286 \\
\hline Glucose & 223 & 221 & 1 & 1 & I \\
\hline Creatinine clearance & 17 & 27 & 80 & 50 & 51 \\
\hline LDH & 369 & 349 & 305 & 1 & I \\
\hline $\mathrm{CK}$ & 2515 & 1735 & 101 & / & l \\
\hline AST & 203 & 136 & 100 & 56 & 21 \\
\hline ALT & 201 & 136 & 106 & 51 & 38 \\
\hline gGT & 398 & 324 & 486 & 530 & 359 \\
\hline ALP & 75 & 117 & 263 & 248 & 155 \\
\hline Total bilirubin & 4.6 & 7.6 & 1.4 & 1.6 & 0.9 \\
\hline CRP & 247 & 225 & 60 & 28 & 8 \\
\hline
\end{tabular}

Hemoglobin $(\mathrm{Hb})$ is expressed in grams per liter; Blood glucose is expressed as milligrams per milliliter; Neutrophil cells, platelets, lymphocytes and monocytes are expressed as $10^{3} / \mathrm{mm}^{3}$; Creatinine clearance is expressed in milliliter per minute and per $1.73 \mathrm{~m}^{2}$; Lactate dehydrogenase (LDH), creatine kinase (CK), aspartate transaminase (AST), alanine transaminase (ALT), gamma-glutamyl transferase (gGT), and alkaline phosphatase (ALP) are all expressed in international units per liter; Total bilirubin and C-reactive protein are expressed in milligrams per deciliter.

\begin{tabular}{|c|c|c|}
\hline Frequency & Organ & Pathology \\
\hline \multirow[t]{2}{*}{ Very frequent $(>1 / 10)$} & Kidney and urinary tract & Cystitis, hematuria, dysuria pollakiuria \\
\hline & General signs and symptoms & Flu-like syndrome, fever, malaise, fatigue \\
\hline \multirow[t]{6}{*}{ Frequent $(>1 / 100,<1 / 10)$} & Blood & Anemia \\
\hline & Lung & Inflammatory pneumonia \\
\hline & Gl tract & Abdominal pain, nausea, vomiting, diarrhea \\
\hline & Skeleton & Arthralgia, arthritis, myalgia \\
\hline & Kidney and urinary tract & $\begin{array}{l}\text { Incontinence, imperious urination, abnormal urinary tests, urinary } \\
\text { infection }\end{array}$ \\
\hline & General signs and symptoms & Chills \\
\hline \multirow[t]{6}{*}{ Sometimes $(>1 / 1000,<1 / 100)$} & Blood & Pancytopenia, thrombocytopenia \\
\hline & Lung & \\
\hline & Gl tract & Hepatitis, increased transaminases \\
\hline & Skeleton & Rash, exanthema \\
\hline & Kidney and urinary tract & Bladder contraction, ureteric obstruction, pyuria, urinary retention \\
\hline & General signs and symptoms & Tuberculosis infections \\
\hline \multirow[t]{2}{*}{$\operatorname{Rare}(>1 / 10000,<1 / 1000)$} & Respiratory tract & Cough \\
\hline & Kidney and urinary tract & Epididymitis \\
\hline \multirow[t]{5}{*}{ Very rare $(<1 / 10000)$} & General signs and symptoms & $\begin{array}{l}\text { Pharyngitis, Reiter syndrome, lupus vulgaris, hypotension, } \\
\text { lymphadenitis }\end{array}$ \\
\hline & Central nervous system & $\begin{array}{l}\text { Anorexia, confusion, dysesthesia, paresthesia, hyperesthesia, } \\
\text { headache, drowsiness }\end{array}$ \\
\hline & Eye & Conjunctivitis \\
\hline & ENT & Vertigo \\
\hline & Kidney and urinary tract & Orchitis \\
\hline
\end{tabular}


are also described and include granulomatous pneumonia, granulomatous hepatitis as well as aspecific dermatologic, ophthalmic, and hematopoietic manifestations.

These systemic disseminations are induced by traumatic catheterization, ${ }^{[1-3]}$ previous instillation, bladder perforation, concomitant radiotherapy, prostate or bladder surgery for previous instillations.

Our patient had none of the local symptoms described previously and developed a systemic BCGitis manifesting by a sepsis followed by a granulomatous pneumonia (lung damage) and possibly liver failure.

The first symptoms marked by a flu-like syndrome can occur immediately after the instillation, which was the case of our patient. This flu like syndrome was consisting of oscillating daily fever peaks ranging from 38 to $39.5^{\circ} \mathrm{C}$.

Our case was unique (to our knowledge) as the first symptom was sepsis of rapid onset requiring admission to ICU, almost never listed in the literature. Sepsis is a rare side effect $(0.4 \%)$ and reported cases show this complication as an immediate consequence of the instillation. ${ }^{[1,3,4]}$

Pulmonary and hepatic manifestations occur normally within an average of two to eight weeks, whereas in our case, the symptoms appeared after 2 weeks had already passed. According to our case report, we can divide the evidences into two categories (Table 2):

In the first place, the non-specific signs such as inflammatory syndrome, ${ }^{[5]}$ increased liver enzymes and lung damage (pulmonary auscultation may reveal a crackling, chest x-ray can show an interstitial syndrome and atelectasis, chest tomography scan determining the precise topography of the lesions) can be observed.

Second, it is possible to spot specific evidences of systemic BCGitis such as cultures of Mycobacteria and PCR in the urines (blood and BAL could all be negative, Ziel and Auramine colorations are generally negative, ${ }^{[5]}$ intradermal reaction is generally negative. ${ }^{[3]}$ If a lung damage is present: histopathology and cytology analysis may reveal the presence of bronchial granulomas (only in $40 \%$ of biopsies). Alcohol-resistant bacilli can be rarely highlighted by coloring (Ziehl or others). The diagnosis is generally acquired through pathology and obtained via bronchial, hepatic or prostatic biopsies showing the presence of granulomas. ${ }^{[5]}$ In many cases, these tests are also negative and it is recommended to start antituberculosis antimicrobials empirically in case of clinical suspicion of the disease. ${ }^{[6]}$ In our case, the patient presented an inflammatory syndrome associated with increased liver enzymes. At the onset of the symptoms, lung auscultation revealed a crinkly. Radiography has highlighted the presence of a basal infiltrate right and the thoracic tomographic scanner made it clear that the lesions were bilateral. The specific enzymatic coloration came back negative. Bronchial biopsies were collected but were not contributive to the diagnosis as showing no evidences of granulomas. Polymerase chain reaction (PCR) has not been carried out on samples.

Literature accepts that the treatment of systemic BCGitis associates tuberculosis antibiotics and corticosteroids even if the treatment with corticosteroids is not universally accepted ${ }^{[1,3,5]}$ Corticosteroid therapy improves the clinical state of the patient ${ }^{[7]}$ and in the case of reported deaths, corticosteroid therapy had not been implemented, ${ }^{[5]}$ but the literature differs on the molecules to be used, their doses, and the duration of therapy. ${ }^{[8-10]}$

\section{CONCLUSIONS}

The systemic BCGitis following an intra-bladder instillation of BCG is a rare complication. The BCGitis should be due to a double reaction, including an immuno-allergic and an bacteremic spread. In our case, it came as severe sepsis in the hours that followed a traumatic instillation. Liver and lung impairments have occurred later. The specific examinations (blood cultures, research of tuberculosis bacillus with color Zielh and other enzymatic colorations, bronchial biopsies) were not contributory but typical aspecific clinical manifestations (syndrome flu-like, fever spikes, and correlated chills) and aspecific biological criteria (increased CRP and increased liver enzymes), had brought us to an empirical treatment and the exclusion diagnosis for systemic BCGitis.

Here, the favoring factor for a bacteriemic release was the instillation after a traumatic act, so it is important to not instillate the BCG if the catheterization is traumatic.

The treatment is based on an extended antituberculous therapy associated with a corticosteroid. The prognosis is generally good under this treatment.

\section{Conflict of interest}

The authors declared no conflicts of interests.

\section{REFERENCES}

1. Gontero P, Bohle A, Malmstrom PU, O’Donnell MA, Oderda M, Sylvester $\mathrm{R}$, et al. The role of bacillus Calmette-Guérin in the treatment of nonmuscle-invasive bladder cancer. Eur Urol 2010; 57: 410-29.

2. Sicard D, Steg A, Leleu C, Boccaccio F, Abadia R, Tulliez M, et al. "BCGitis", a systemic complication of intravesical BCG therapy of bladder 
tumor. Ann Med Int 1987; 138: 555-6.

3. Demers V, Pelsser V. "BCGitis": A rare case of tuberculous epididymoorchitis following intravesical Bacillus Calmette-Guérin therapy. J Radiol Case Rep 2012; 6: 16-21.

4. Rival G, Garodt D, Mercier E, Narciso B, Legras A, Perrotin D, et al. Acute respiratory failure and septic shock induced by Mycobacterium bovis. A rare side effect of intravesical BCG therapy. Press Med 2006; 35: 36-42.

5. de Saint Martin L, Boiron C, Poveda JD, Herreman G. Generalized BCG infection after intravesical instillations of Calmette-Guerin bacillus. Press Med 1993; 22: 1352-6.

6. Elkabani M, Greene JN, Vincent AL, VanHook S, Sandin RL. Disseminated Mycobacterium bovis after intravesicular bacillus calmette-Guerin treatments for bladder cancer. Cancer Control 2000; 7: 476-81.

7. Wittes R, Klotz L, Kosecka U. Severe bacillus Calmette-Guerin cystitis responds to systemic steroids when antituberculous drugs and local steroids fail. J Urol 1999; 161: 1568-9.
8. Lamm DJ. Complications of Bacillus Calmette-Guerin Immunotherapy. Urol Clin North Am 1992; 19: 565-72.

9. Caulier P, Yombi JC, Dufaux M, Feyaerts A, Abi AA, Hainaut P. Prostate abcesses following intravescical BCG therapy. Acta Clin Belg 2009; 64: 436-7.

10. McParland C, Cotton DJ, Gowda KS, Hoeppner VH, Martin WT, Weckworth PF. Miliary Mycobacterium bovis induced by intravesical bacille Calmette-Guérin immunotherapy. Am Rev Respir Dis 1992; 146: 1330-3.

How to cite this article: Attou R, Albrich T, Kadou J, Redant S, Honoré PM, De Bels D. Favorable outcome in a patient with systemic BCGitis after intra-bladder instillation of Calmette-Guerin Bacillus highlighting the importance of making the correct diagnosis in this rare form of sepsis. J Transl Int Med 2019; 7: 34-8. 